\title{
In Vitro Identification of Hamiltonian Cycle using Circular Structure Assisted DNA Computer
}

Deepak Sharma and Manojkumar Ramteke*广

$†$ Department of Chemical Engineering, Indian Institute of Technology Delhi, New Delhi, 110016, INDIA

*E-mail: mcramteke@chemical.iitd.ac.in,ramtekemanoj@gmail.com

\section{Supporting Information}

Table S1. Enzymes and the DNA sequences* used for vertices for Problem 1 (6 bp sequences at the $5^{\prime}$ end of the vertices is the recognition site for the respective enzyme).

\begin{tabular}{lll}
\hline Enzymes & Vertices & Sequences $\left(5^{\prime} \rightarrow 3^{`}\right)$ \\
\hline AanI (PsiI) & $V_{1}$ & TTATAACCGGCGTGAC \\
Ajil (BmgBI) & $V_{2}$ & CACGTCGAGTTGATAC \\
Bsp68I (NruI) & $V_{3}$ & TCGCGAGTATACTACG \\
PvuII & $V_{4}$ & CAGCTGATAGTGACTC \\
Bst1107I (BstZ17I) & $V_{5}$ & GTATACACTAGCTGGC \\
NsbI (FspI) & $V_{6}$ & TGCGCAATGGACTTAC \\
DraI & $V_{7}$ & TTTAAACGCGGTGCCA \\
Ec1136II (EcoICRI) & $V_{8}$ & GAGCTCGATCCAGATT \\
Eco105I (SnaBI) & $V_{9}$ & TACGTATCAGGCCTAG
\end{tabular}




$\begin{array}{lcc}\text { Eco147I (StuI) } & V_{10} & \text { AGGCCTAGACTCTGAT } \\ \text { Eco32I (EcoRV) } & V_{11} & \text { GATATCCGACGTCAGT } \\ \text { Eco47III (AfeI) } & V_{12} & \text { AGCGCTCGTATATCAG } \\ \text { Eco72I (PmlI) } & V_{13} & \text { CACGTGATGGATCTCA } \\ \text { EheI (SfoI) } & V_{14} & \text { GGCGCCTAGATCTTAA } \\ \text { ScaI } & V_{15} & \text { AGTACTTAGTCGCGCA } \\ \text { KspAI (HpaI) } & V_{16} & \text { GTTAACAACGTCGGTC } \\ \text { MlsI (MscI) } & V_{17} & \text { TGGCCAACACGTGTAT } \\ \text { SspI } & V_{18} & \text { AATATTGGTCGCCGAC }\end{array}$

*All the oligonucleotide sequences and primers are synthesized by Xcelris Labs Limited, Ahmedabad, Gujarat, India using desalted purification method.

Table S2. DNA sequences* for the edges used to solve Problem 1.

\begin{tabular}{ll}
\hline Edges & Sequences $\left(5^{`} \rightarrow 3^{`}\right)$ \\
\hline$E_{1 \rightarrow 13}$ & ATCACGTGGTCACGCC \\
$E_{2 \rightarrow 3}$ & ACTCGCGAGTATCAAC \\
$E_{2 \rightarrow 8}$ & TCGAGCTCGTATCAAC \\
$E_{2 \rightarrow 11}$ & CGGATATCGTATCAAC \\
$E_{3 \rightarrow 7}$ & CGTTTAAACGTAGTAT \\
$E_{3 \rightarrow 9}$ & GATACGTACGTAGTAT \\
$E_{4 \rightarrow 5}$ & GTGTATACGAGTCACT \\
$E_{4 \rightarrow 17}$ & GTTGGCCAGAGTCACT \\
$E_{5 \rightarrow 8}$ & TCGAGCTCGCCAGCTA \\
$E_{6 \rightarrow 1}$ & GGTTATAAGTAAGTCC \\
$E_{6 \rightarrow 13}$ & ATCACGTGGTAAGTCC \\
$E_{6 \rightarrow 15}$ & TAAGTACTGTAAGTCC \\
$E_{7 \rightarrow 16}$ & TTGTTAACTGGCACCG
\end{tabular}




\begin{tabular}{ll}
$E_{7 \rightarrow 18}$ & CCAATATTTGGCACCG \\
$E_{8 \rightarrow 12}$ & CGAGCGCTAATCTGGA \\
$E_{9 \rightarrow 17}$ & GTTGGCCACTAGGCCT \\
$E_{10 \rightarrow 6}$ & ATTGCGCAATCAGAGT \\
$E_{10 \rightarrow 15}$ & TAAGTACTATCAGAGT \\
$E_{11 \rightarrow 4}$ & ATCAGCTGACTGACGT \\
$E_{11 \rightarrow 16}$ & TTGTTAACACTGACGT \\
$E_{12 \rightarrow 8}$ & TCGAGCTCCTGATATA \\
$E_{12 \rightarrow 11}$ & CGGATATCCTGATATA \\
$E_{13 \rightarrow 9}$ & GATACGTATGAGATCC \\
$E_{14 \rightarrow 5}$ & GTGTATACTTAAGATC \\
$E_{14 \rightarrow 10}$ & CTAGGCCTTTAAGATC \\
$E_{15 \rightarrow 4}$ & ATCAGCTGTGCGCGAC \\
$E_{15 \rightarrow 17}$ & GTTGGCCATGCGCGAC \\
$E_{16 \rightarrow 2}$ & TCGACGTGGACCGACG \\
$E_{16 \rightarrow 5}$ & GTGTATACGACCGACG \\
$E_{17 \rightarrow 15}$ & TAAGACTATACACGT \\
$E_{18 \rightarrow 14}$ & TAGGCCGTCGGCGA \\
\hline &
\end{tabular}

*All the oligonucleotide sequences are synthesized by Xcelris Labs Limited, Ahmedabad, Gujarat, India using desalted purification method. 
Table S3: Operating conditions used to solve the Hamiltonian cycle.

\begin{tabular}{|c|c|c|c|c|c|}
\hline $\begin{array}{l}\text { Sr. } \\
\text { No. }\end{array}$ & Process & $\begin{array}{l}\text { Temperature } \\
\left({ }^{\circ} \mathrm{C}\right)\end{array}$ & Time & Enzyme & $\begin{array}{l}\text { Concentrations } \\
\text { used }\end{array}$ \\
\hline 1. & $\begin{array}{l}\text { Gel } \\
\text { electrophoresis }\end{array}$ & $22-25$ & $\begin{array}{l}60-90 \\
(\min )\end{array}$ & - & $\begin{array}{lll}\text { Agarose: } & 2-3 \quad \% \\
\mathrm{w} / \mathrm{v} & & \end{array}$ \\
\hline 2. & Ligation & 37 & $60(\mathrm{~min})$ & DNA Ligase & $\begin{array}{l}\text { Each oligo: } 50 \\
\text { pmol } \\
\text { T4 DNA ligase: } \\
0.5 \mu 1 \\
10 \mathrm{X} \text { Ligase } \\
\text { buffer: } 10 \mu 1 \\
\text { Nuclease free } \\
\text { water: to } 100 \mu 1 \\
\end{array}$ \\
\hline 3. & PCR (one & $91-93$ & $15(\mathrm{sec})$ & DNA Taq & $\begin{array}{l}\text { Forward primer: } \\
50 \mathrm{pmol}\end{array}$ \\
\hline & cycle) & $50-52$ & $\begin{array}{l}20-30 \\
(\mathrm{sec})\end{array}$ & polymerase & $\begin{array}{l}\text { Reverse primer: } \\
50 \text { pmol } \\
\text { Taq DNA } \\
\text { polymerase: } 0.5 \mu 1 \\
10 \text { X PCR buffer: }\end{array}$ \\
\hline & & 72 & $\begin{array}{l}20-30 \\
(\mathrm{sec})\end{array}$ & & $\begin{array}{l}5 \mu 1 \\
\text { Nuclease free } \\
\text { water: to } 50 \mu 1\end{array}$ \\
\hline \multirow[t]{2}{*}{4.} & \multirow[t]{2}{*}{ DNA digestion } & 37 & $60 \mathrm{~min}$ & $\begin{array}{l}\text { Restriction enzyme } \\
\text { corresponding to } \\
\text { vertex (see Table S1, } \\
\text { S3, and S5) }\end{array}$ & \multirow[t]{2}{*}{$\begin{array}{l}\text { PCR mixture: } 10 \\
\mu 1 \\
\text { Nuclease free } \\
\text { water: } 18 \mu 1 \\
10 \mathrm{X} \text { buffer: } 2 \mu 1 \\
\text { Restriction } \\
\text { enzyme: } 1-2 \mu 1\end{array}$} \\
\hline & & $\begin{array}{l}65 \quad \text { (heat } \\
\text { inactivation) }\end{array}$ & $\begin{array}{l}15-20 \\
\min \end{array}$ & & \\
\hline
\end{tabular}


Table S4: Detailed procedure for extracting the DNA from the gel.

1. Mark the position of gel slice in the gel for the given DNA size to be extracted.

2. Cut the gel slice by using a blade and transfer the gel slice into a tube.

3. Fill the tube with the elution buffer till the gel slice is dipped completely.

4. Heat the tube at $65^{\circ} \mathrm{C}$ to melt the slice of gel.

5. Place the tube with melted gel at $-70{ }^{\circ} \mathrm{C}$ for 10 mins.

6. Centrifuge the frozen gel in the tube for $10 \mathrm{~min}$ and transfer the supernatant to another tube.

7. Repeat the steps from $3-6$, for three times.

8. Add n-butanol to the tube of supernatant and mix the content.

9. The tube is then put into a vortex shaker for $15 \mathrm{~min}$ to remove the ethidium bromide added during the gel formation.

10. Remove the layer of butanol and ethidium bromide formed during step 9 and repeat the process for two times.

11. Add ethanol in the tube, mix the content and keep at $-70{ }^{\circ} \mathrm{C}$ for $30 \mathrm{~min}$.

12. Centrifuge the mixture in the tube for $15 \mathrm{~min}$ and discard the supernatant.

13. Add $150-200 \mu \mathrm{l}$ ethanol (70\%) into the tube, centrifuge for $5 \mathrm{~min}$ and remove the supernatant.

14. The obtained DNA solution after step 13 is added with TE (Tris and EDTA) buffer and used for further process.

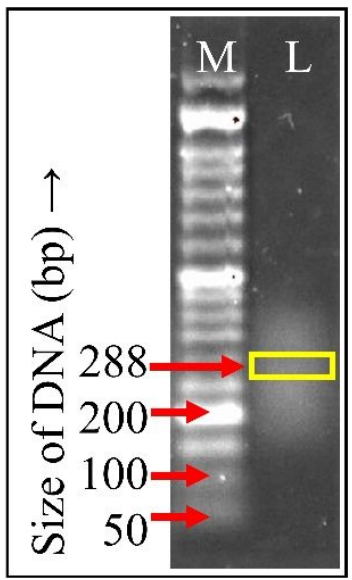

Figure S1: Agarose gel electrophoresis image (Lane L) after step (c) (ligation) for Problem 1. Lane $\mathrm{M}$ shows the marker of $50 \mathrm{bp}$. 


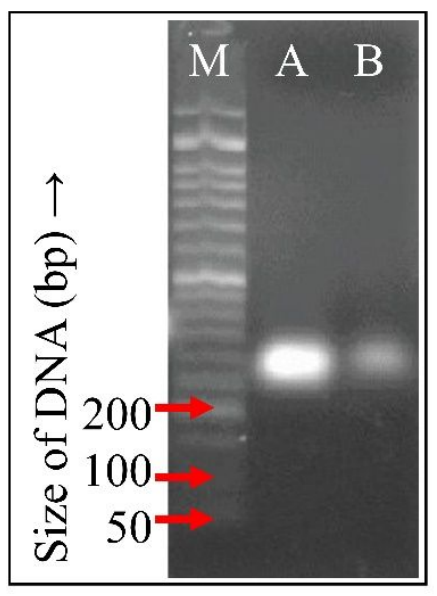

Figure S2: Agarose gel electrophoresis results after and before step (e) (selective PCR and magnetic bead separation) are shown as lane A and lane B for Problem 1. Darker band in lane A illustrates the selective amplification of the DNA solutions. Lane M shows the marker of $50 \mathrm{bp}$.

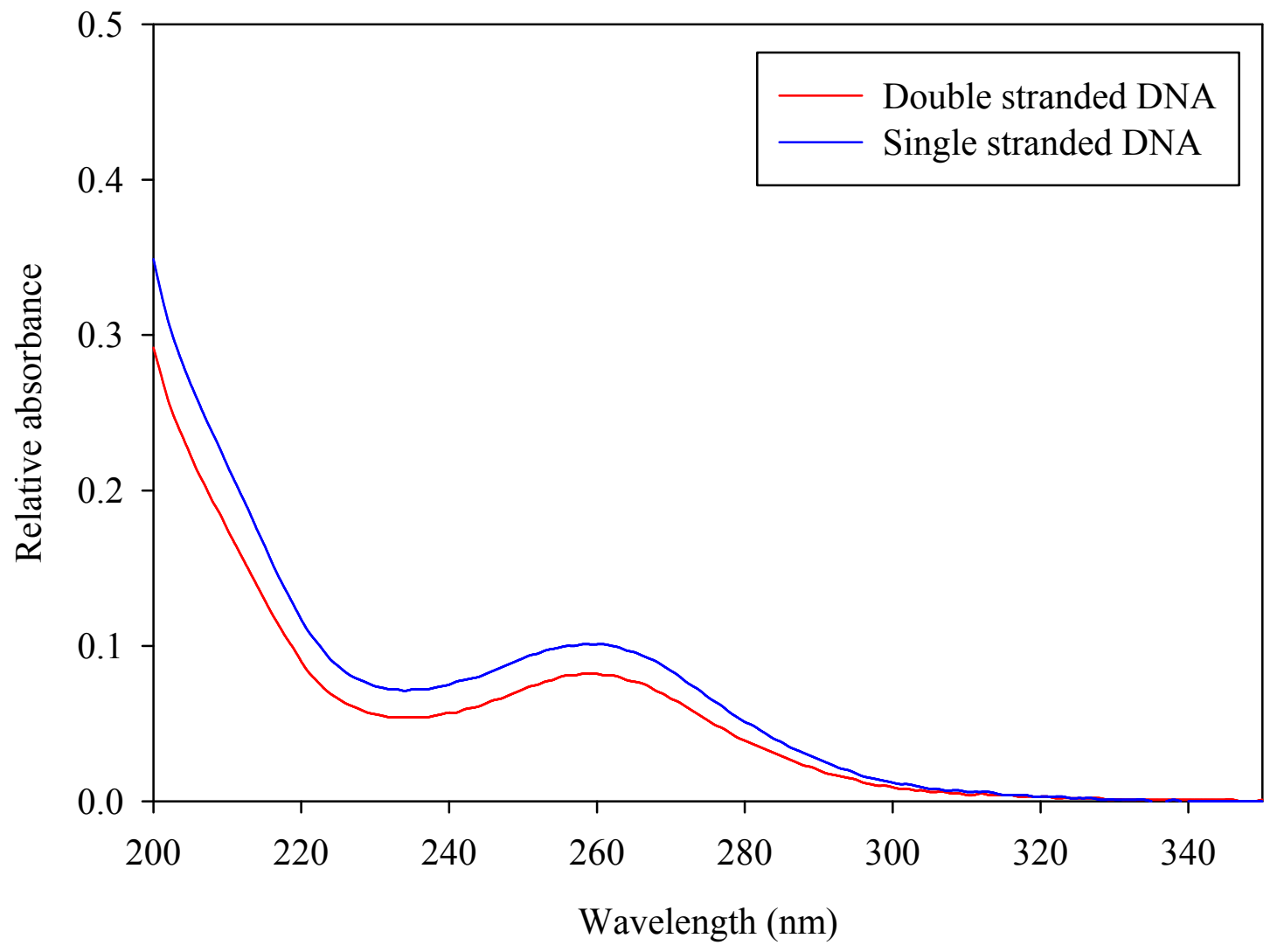

Figure S3: Absorbance curve (UV spectroscopy) of DNA solutions after step (e) (selective PCR and magnetic bead separation) and after step (f) circularization illustrating the presence of single and double-stranded DNA, respectively. 


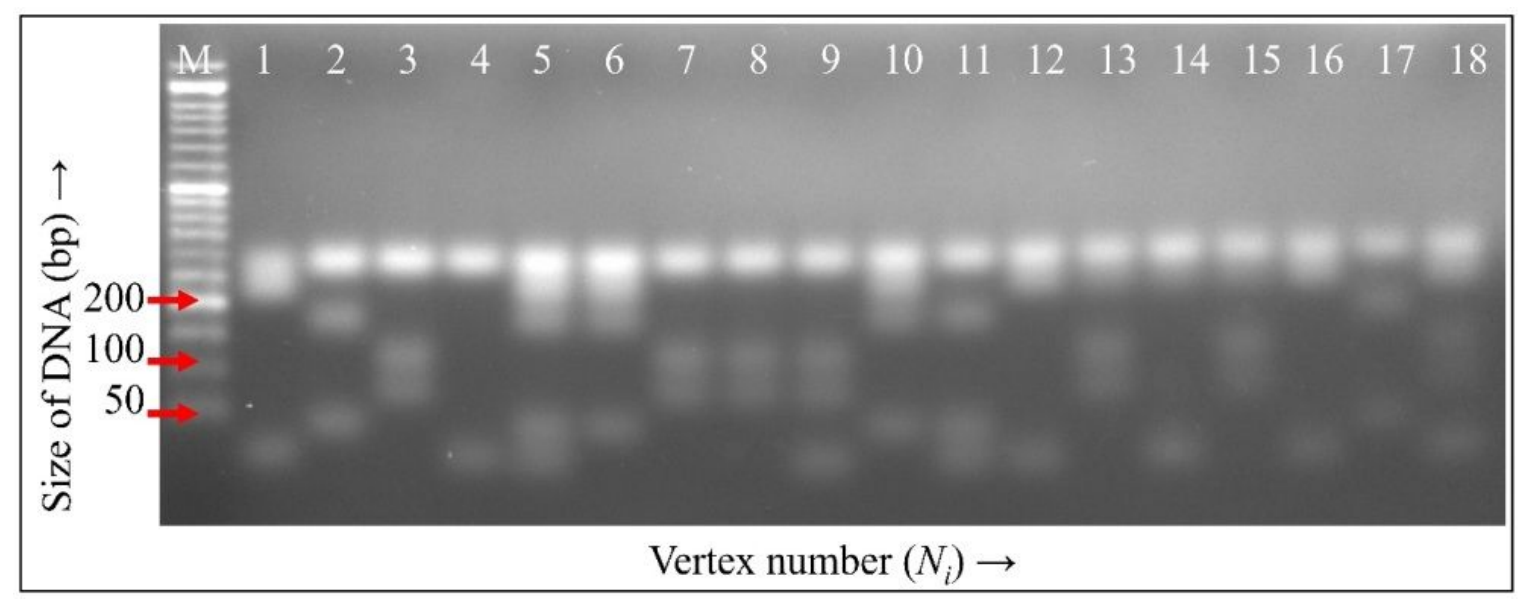

Figure S4. The intermediate gel electrophoresis images representing the DNA bands obtained after each (g) digestion step (corresponding to $1-18$ vertices) of the DNA with restriction enzymes and before step (d) for Problem 1. The correct DNA solution in each lane (corresponding to $1-18$ vertices) corresponds to the size of 288 base pair. Multiple bands present in the same lane are due to the presence of the vertex $V_{i}$ in respective solution at multiple positions. Except for the DNA corresponding to $288 \mathrm{bp}$ band, all other DNA solutions are eliminated in (d) gel electrophoresis operation performed after each restriction enzyme digestion step to give Hamiltonian cycle after the end of the $18^{\text {th }}$ step. The DNA solution obtained after the $18^{\text {th }}$ step is subjected to the second part of the procedure to give Figure 4 .

Table S5. Enzymes and the DNA sequences* used for vertices for Problem 2 (6 bp sequences at the $5^{\prime}$ end of the vertices is the recognition site for the respective enzyme).

\begin{tabular}{lll}
\hline Enzymes & Vertices & Sequences $\left(5^{`} \rightarrow 3^{`}\right)$ \\
\hline Bst1107I (BstZ17I) & $V_{1}$ & GTATACTCTGTCACTAGGCTGCGAACTCAAGG \\
Eco105I $($ SnaBI $)$ & $V_{2}$ & TACGTACTATCATGGTCACAGGCGGATCTCAG
\end{tabular}




\begin{tabular}{lll} 
Bsp68I (NruI) & $V_{3}$ & TCGCGATCTCAGAGTCAGTCCAGTAGTAAGTC \\
Eco72I (PmlI) & $V_{4}$ & CACGTGCAGTATCCGGAGAGTGTTCACATATC \\
AanI (PsiI) & $V_{5}$ & TTATAAGGCTCGTCCGATCGCACAGTTCAGGA \\
Eco147I (StuI) & $V_{6}$ & AGGCCTAGTCTACCGGTTCCATAGATCAGTGA \\
Ecl136II (EcoICRI) & $V_{7}$ & GAGCTCATATGACCGGTAGAACTGTAGCCTCT \\
EheI (SfoI) & $V_{8}$ & GGCGCCTAGATCTTAAGCTACAGTGTACCATG \\
MlsI (MscI) & $V_{9}$ & TGGCCAACACGTGTATGATCAGCTGTCCATAG \\
SspI & $V_{10}$ & AATATTGGCATAGAGTCTCGCTACGTCGCAGC \\
\hline
\end{tabular}

*All the oligonucleotide sequences and primers are synthesized by Xcelris Labs Limited, Ahmedabad, Gujarat, India using desalted purification method.

Table S6. DNA sequences* for the edges used to solve Problem 2.

\begin{tabular}{ll}
\hline Edges & Sequences $\left(5^{`} \rightarrow 3^{`}\right)$ \\
\hline$E_{l \rightarrow 2}$ & ACCATGATAGTACGTACCTTGAGTTCGCAGCC \\
$E_{2 \rightarrow 8}$ & TTAAGATCTAGGCGCCCTGAGATCCGCCTGTG \\
$E_{3 \rightarrow 1}$ & TAGTGACAGAGTATACGACTTACTACTGGACT \\
$E_{3 \rightarrow 6}$ & CCGGTAGACTAGGCCTGACTTACTACTGGACT \\
$E_{4 \rightarrow 1}$ & TAGTGACAGAGTATACGATATGTGAACACTCT \\
$E_{4 \rightarrow 8}$ & TTAAGATCTAGGCGCCGATATGTGAACACTCT \\
$E_{5 \rightarrow 4}$ & CCGGATACTGCACGTGTCCTGAACTGTGCGAT \\
$E_{5 \rightarrow 9}$ & ATACACGTGTTGGCCATCCTGAACTGTGCGAT \\
$E_{6 \rightarrow 2}$ & ACCATGATAGTACGTATCACTGATCTATGGAA \\
$E_{6 \rightarrow 10}$ & ACTCTATGCCAATATTTCACTGATCTATGGAA \\
$E_{7 \rightarrow 1}$ & TAGTGACAGAGTATACAGAGGCTACAGTTCTA \\
$E_{7 \rightarrow 5}$ & CGGACGAGCCTTATAAAGAGGCTACAGTTCTA \\
$E_{8 \rightarrow 2}$ & ACCATGATAGTACGTACATGGTACACTGTAGC \\
$E_{8 \rightarrow 3}$ & GACTCTGAGATCGCGACATGGTACACTGTAGC
\end{tabular}




$$
\begin{array}{ll}
E_{9 \rightarrow 2} & \text { ACCATGATAGTACGTACTATGGACAGCTGATC } \\
E_{9 \rightarrow 8} & \text { TTAAGATCTAGGCGCCCTATGGACAGCTGATC } \\
E_{10 \rightarrow 1} & \text { TAGTGACAGAGTATACGCTGCGACGTAGCGAG } \\
E_{10 \rightarrow 6} & \text { CCGGTAGACTAGGCCTGCTGCGACGTAGCGAG } \\
E_{10 \rightarrow 9} & \text { ATACACGTGTTGGCCAGCTGCGACGTAGCGAG }
\end{array}
$$

*All the oligonucleotide sequences are synthesized by Xcelris Labs Limited, Ahmedabad, Gujarat, India using desalted purification method.

Table S7. Enzymes and the DNA sequences* used for vertices for Problem 3 (6 bp sequences at the $5^{\prime}$ end of the vertices is the recognition site for the respective enzyme).

\begin{tabular}{lll}
\hline Enzymes & Vertices & Sequences $\left(5^{`} \rightarrow 3^{`}\right)$ \\
\hline Bst1107I (BstZ17I) & $V_{1}$ & GTATACTCTGTCACTAGGCTGCGAACTCAAGG \\
Eco105I (SnaBI) & $V_{2}$ & TACGTACTATCATGGTCACAGGCGGATCTCAG \\
Bsp68I (NruI) & $V_{3}$ & TCGCGATCTCAGAGTCAGTCCAGTAGTAAGTC \\
Eco72I (PmlI) & $V_{4}$ & CACGTGCAGTATCCGGAGAGTGTTCACATATC \\
AanI (PsiI) & $V_{5}$ & TTATAAGGCTCGTCCGATCGCACAGTTCAGGA \\
Eco147I (StuI) & $V_{6}$ & AGGCCTAGTCTACCGGTTCCATAGATCAGTGA \\
Ec1136II (EcoICRI) & $V_{7}$ & GAGCTCATATGACCGGTAGAACTGTAGCCTCT
\end{tabular}

*All the oligonucleotide sequences and primers are synthesized by Xcelris Labs Limited, Ahmedabad, Gujarat, India using desalted purification method.

Table S8. DNA sequences* for the edges used to solve Problem 3.

\begin{tabular}{ll}
\hline Edges & Sequences $\left(5^{`} \rightarrow 3^{`}\right)$ \\
\hline$E_{1 \rightarrow 6}$ & CCGGTAGACTAGGCCTCCTTGAGTTCGCAGCC \\
$E_{1 \rightarrow 7}$ & CCGGTCATATGAGCTCCCTTGAGTTCGCAGCC \\
$E_{2 \rightarrow 1}$ & TAGTGACAGAGTATACCTGAGATCCGCCTGTG \\
$E_{2 \rightarrow 5}$ & CGGACGAGCCTTATAACTGAGATCCGCCTGTG
\end{tabular}




\begin{tabular}{ll}
$E_{2 \rightarrow 6}$ & CCGGTAGACTAGGCCTCTGAGATCCGCCTGTG \\
$E_{2 \rightarrow 7}$ & CCGGTCATATGAGCTCCTGAGATCCGCCTGTG \\
$E_{3 \rightarrow 4}$ & CCGGATACTGCACGTGGACTTACTACTGGACT \\
$E_{3 \rightarrow 7}$ & CCGGTCATATGAGCTCGACTTACTACTGGACT \\
$E_{4 \rightarrow 3}$ & GACTCTGAGATCGCGAGATATGTGAACACTCT \\
$E_{4 \rightarrow 5}$ & CGGACGAGCCTTATAAGATATGTGAACACTCT \\
$E_{5 \rightarrow 4}$ & CCGGATACTGCACGTGTCCTGAACTGTGCGAT \\
$E_{6 \rightarrow 1}$ & TAGTGACAGAGTATACTCACTGATCTATGGAA \\
$E_{6 \rightarrow 2}$ & ACCATGATAGTACGTATCACTGATCTATGGAA \\
$E_{6 \rightarrow 7}$ & CCGGTCATATGAGCTCTCACTGATCTATGGAA \\
$E_{7 \rightarrow 1}$ & TAGTGACAGAGTATACAGAGGCTACAGTTCTA \\
$E_{7 \rightarrow 2}$ & ACCATGATAGTACGTAAGAGGCTACAGTTCTA \\
$E_{7 \rightarrow 3}$ & GACTCTGAGATCGCGAAGAGGCTACAGTTCTA \\
$E_{7 \rightarrow 4}$ & CCGGATACTGCACGTGAGAGGCTACAGTTCTA \\
$E_{7 \rightarrow 6}$ & CCGGTAGACTAGGCCTAGAGGCTACAGTTCTA \\
\hline
\end{tabular}

*All the oligonucleotide sequences are synthesized by Xcelris Labs Limited, Ahmedabad, Gujarat, India using desalted purification method. 